\title{
Biodiversidad de macrocrustáceos del río Fuerte, Sinaloa, México
}

\section{Biodiversity of macrocrustaceans of river Fuerte, Sinaloa, Mexico}

\author{
Carlos M. Valdez-Flores ${ }^{1}$, Geovany Soto-Domínguez ${ }^{1}$, Pedro Hernández-Sandoval ${ }^{1}$, \\ Jesús G. Luna-Valdez ${ }^{2}$, Gerardo Rangel-Cota ${ }^{3}$ y Marcelo U. García Guerrero ${ }^{4}$
}

'Unidad Regional Los Mochis de la Universidad Autónoma de Occidente, Departamento de Ciencias Naturales y Exactas, Boulevard Macario Gaxiola s/n, CP 81217, Los Mochis, Sinaloa, México.

2 Unidad Regional Los Mochis de la Universidad Autónoma de Occidente. Departamento Académico de Ingeniería y Tecnología. Boulevard Macario Gaxiola s/n, CP. 81217. Los Mochis, Sinaloa, México.

${ }^{3}$ Instituto Tecnológico de Los Mochis. Licenciatura en Biología. Boulevard Juan de Dios Bátiz y 20 de noviembre. A.P. 766. Los Mochis, Sinaloa, México.

${ }^{4}$ Laboratorio de Acuacultura, CIIDIR-IPN Oaxaca, Calle Hornos 1003, Col. Noche Buena, Santa Cruz Xoxocotlán, Oaxaca, 71230. México.

E-mail: pedro.hernandez@uadeo.mx

RESUMEN. El río Fuerte, a través de su cauce forma grandes ecosistemas que sirven de hábitat para especies de macrocrustáceos de agua dulce que son de importancia ecológica y económica en la zona norte del estado de Sinaloa, México. El objetivo de esta investigación es generar información acerca de la diversidad de macrocrustáceos y su distribución espacial en el río Fuerte, ya que no existe información publicada al respecto. Se establecieron cinco estaciones de muestreo (El Fuerte, San Blas, San Miguel, San José y Las Grullas) a lo largo de su cauce en función de la altitud (m s.n.m) y distancia entre las mismas, iniciando en el municipio de El Fuerte (EI) hasta su desembocadura en la población de Las Grullas (EV) del municipio de Ahome. La riqueza específica total fue de 12 taxa. Palémonidos y cambáridos fueron los taxa mejor representados. La diversidad (índice de Shannon-Wiener) fue mayor $\left(H^{\prime}=0,92\right)$ respecto a otros ríos de México. El análisis de ordenación nMDS de Bray Curtis indicó que la comunidad se puede agrupar en tres grupos bien definidos. Macrobrachium tenellum y Potimirim glabra fueron las especies más abundantes. El sector Las Grullas tuvo el menor número de individuos, con presencia de especies como Litopenaeus vannamei y Callinectes arcuatus. Además, se registró la presencia de una especie exótica (Procambarus clarkii) y una no citada previamente para la zona ( $P$. g/abra).

Palabras clave: Comunidad, índice ecológico, palémonidos, taxones.

ABSTRACT. The Fuerte river forms large ecosystems in its channel that serve as the habitats for freshwater macrocrustaceans with ecological and economical importance in the Northern area of the state of Sinaloa, Mexico. The objective of this investigation is to generate information about the biodiversity and the spatial patterns of the crustacean community due to the absence of published data of this fauna. Therefore, five sampling stations were established (El Fuerte, San Blas, San Miguel, San José and Las Grullas) along the river channel at different altitudes ( $m$ a.s.l.) and distances, beginning at El Fuerte (EI) municipality until its mouth at Las Grullas (EV) in the Ahome municipality. The specific richness was $12(\mathrm{~S}=12)$. Palaemonids and cambarids were the best represented taxa. The diversity (Shannon-Wiener index) was higher $\left(H^{\prime}=0.92\right)$ than the recorded in other Mexican rivers. The Bray Curtis ordination (nMDS) analysis showed that the community was formed by three well defined groups. Macrobrachium tenellum and Potimirin glabra were the most 
abundant species. Las Grullas sector showed the minor number of individuals, with the presence of species such as Litopenaeus vannamei and Callinectes arcuatus. Furthermore, an exotic species (Procambarus clarkii) was found as well as a species (P. glabra) previously not reported for the region.

Keywords: Community, diversity index, palaemonids, taxa.

\section{INTRODUCCIÓN}

México cuenta con 135 ríos principales, los cuales se encuentran agrupados en tres vertientes (CONAGUA, 2016). Los situados en el estado de Sinaloa pertenecen a la vertiente occidental, es decir, vierten sus aguas al océano Pacífico. Existen 11 ríos, divididos en dos regiones hidrológicas mantenidas gracias a los escurrimientos superficiales provenientes de la sierra de Chihuahua y Durango (INEGI, 2017). El río Fuerte se destaca entre estos ríos. Se ubica en la parte norte del estado, y atraviesa los municipios de El Fuerte y Ahome, formando una de las cuencas más grandes de la entidad y junto con la del Río Sinaloa conforman alrededor del 50\% del total del área hidrológica (Orrantia Verduzco, 2019).

El río Fuerte forma grandes ecosistemas que sirven de hábitat para especies tan heterogéneas como los crustáceos y que representan interés ecológico y económico para el estado. Posiblemente, ningún otro grupo de plantas o animales presentan una diversidad morfológica tan amplia como los crustáceos (Martin \& Davis, 2001). Son tan diversos que sus dimensiones oscilan entre un milímetro y cuatro metros de longitud (Simões et al., 2004). El conocimiento biológico acerca de los crustáceos es más favorable en aquellas especies de interés comercial tales como jaibas, camarones, langostinos y langostas ya que son para consumo humano (García-Madrigal et al., 2012). Sin embargo, la relevancia del taxón, no sólo es alimenticia, sino también ecológica, farmacéutica, entre otras (Bjerkeng, 2000, Marmol et al., 2011). Los crustáceos son fundamentales para el funcionamiento adecuado de los ecosistemas acuáticos, ya que por su abundancia y diversidad proporcionan estructura a las comunidades de macroinvertebrados que habitan en ellos. Además, son consumidores secundarios que se encargan de transformarla materia orgánica en energía y proteínas consumibles por los eslabones finales; también sirven como indicadores de calidad del agua (Álvarez et al., 1996) y constituyen uno de los recursos de mayor importancia dentro de las pesquerías mundiales. En el ambiente dulceacuícola de México, los crustáceos integran un grupo complejo en el cual es posible distinguir formas de varios componentes zoogeográficos; los más característicos son los relacionados con las regiones Neártica y Neotropical (Villalobos-Hiriart et al., 1993). Entre decápodos dulceacuícolas, se han registrado alrededor de 178 especies que se distribuyen en altitudes mayores a los 3000 m s.n.m. (Álvarez et al., 2014; Villalobos et al., 2010). Otros crustáceos de agua dulce y terrestres han sido menos estudiados (Rodríguez-Almaraz et al., 2014). Este desconocimiento del grupo y la acelerada pérdida de hábitat promueve disminución de la diversidad a pesar de que las especies endémicas tienen especial atención en estos hábitats ya que representan más del $80 \%$ para México (Villalobos-Hiriart y Álvarez, 2008). De acuerdo con lo señalado previamente y a la escasa información sobre los crustáceos de agua dulce que habitan el río Fuerte en la zona norte de Sinaloa, se planteó hacer una investigación para determinar la biodiversidad y patrones espaciales de la comunidad de macrocrustáceos, así como actualizar el conocimiento de este grupo en el área de estudio y contribuir al conocimiento de la biodiversidad del estado de Sinaloa.

\section{MATERIALES Y MÉTODOS Área de estudio}

El río Fuerte se ubica en la zona Norte de Sinaloa, México. Se eligieron cinco estaciones de muestreo (Figura 1): El Fuerte (El: 26²4'49.36" $\mathrm{N}, 108^{\circ} 37^{\prime} 45.82^{\prime \prime} \mathrm{O}$ ), San Blas (Ell: $26^{\circ} 4^{\prime} 12.60^{\prime \prime} \mathrm{N}$ 


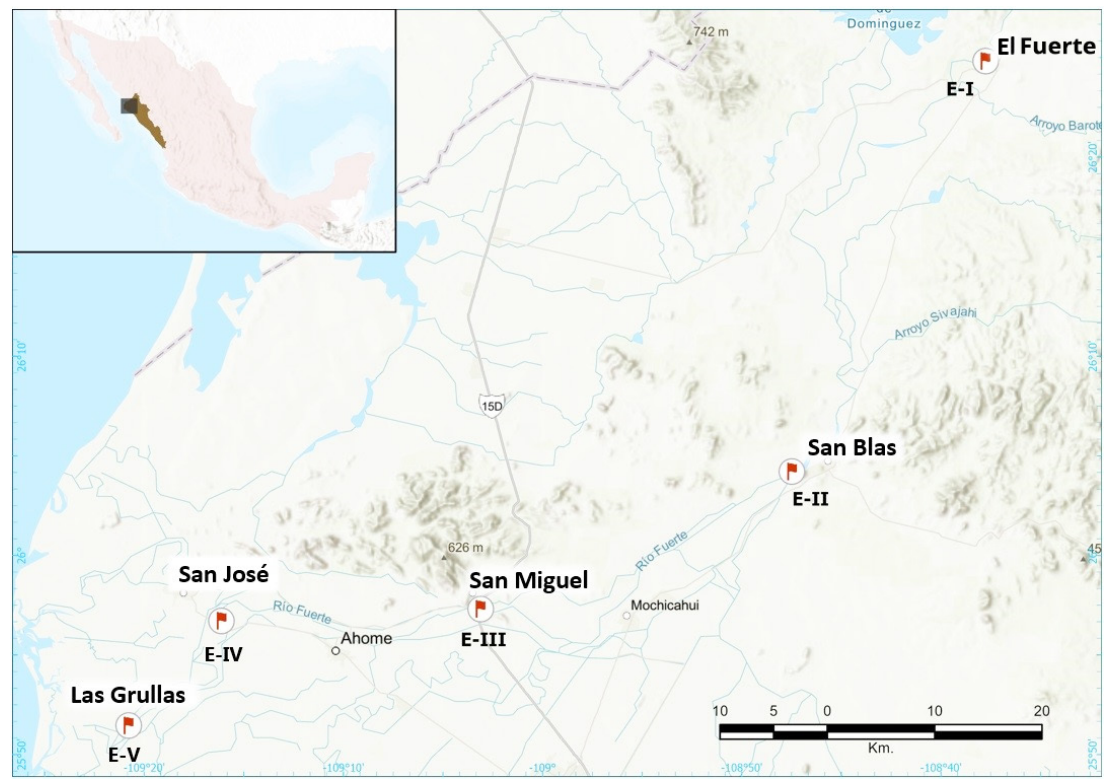

Figura 1. Localización geográfica del Río Fuerte (Sinaloa, México) y de las estaciones de muestreo establecidas.

Figure 1. Geographical location of the Río Fuerte (Sinaloa, Mexico) and the established sampling stations.

108 47'28.50" O), San Miguel (EIII: 255'18.75" N, $109^{\circ} 3^{\prime} 6.67^{\prime \prime}$ O), San José (EIV: 2556'43.14" N, $109^{\circ} 16^{\prime} 6.81^{\prime \prime} \mathrm{O}$ ) y Las Grullas (EV: $25^{\circ} 51^{\prime} 27.68^{\prime \prime}$ $\left.\mathrm{N}, 109^{\circ} 20^{\prime} 46.90^{\prime \prime} \mathrm{O}\right)$. La longitud del cauce estudiado fue de $231 \mathrm{~km}$ y la mayor altitud registrada fue de $165 \mathrm{~m}$ s.n.m.

\section{Trabajo de campo}

Se realizaron muestreos mensuales durante el período mayo-octubre de 2017. En cada sitio se realizaron tres arrastres con chinchorro playero de $10 \mathrm{~m}$ de longitud, $1,5 \mathrm{~m}$ de alto y $0,5 \mathrm{~mm}$ de abertura de malla. Los organismos colectados se colocaron en bolsas de plástico dentro de un contenedor con hielo para su trasladado al laboratorio de Química de la Universidad Autónoma de Occidente, Unidad Regional Los Mochis, donde fueron depositados en frascos con formol (Formalina 4\%). Después de una semana se transfirieron a alcohol al 70\% para su posterior identificación.

\section{Análisis de la biodiversidad}

Para el análisis de la biodiversidad y los patrones comunitarios de crustáceos se utilizaron la riqueza específica (S) mediante la identificación y recuento de las especies presentes y la abundancia relativa que representa un valor ponderado del número de organismos de cada especie. En cada sitio de colecta se calculó la diversidad, dominancia y equidad mediante los índices de Shannon-Wiener $\left(\mathrm{H}^{\prime}\right)$, Simpson (C) y Pielou (J') (Baev \& Penev, 1995; Magurran, 1988; Moreno, 2001), respectivamente. Además, se efectuó el análisis de escalamiento multidimensional no métrico (nMDS) basado en análisis de similaridad de Bray-Curtis y análisis de varianza multivariado, con base en permutaciones (PERMANOVA) para determinar si existen diferencias significativas entre la abundancia de crustáceos. Para los análisis se utilizó el software PRIMER-E v6 (Araujo-Leyva et al., 2020) y los datos de abundancia de las especies fueron transformados mediante raíz cuadrada. Para el registro y manejo de datos se empleó el software Microsoft Excel 2010๔, y para determinar los índices ecológicos se utilizó el softwarepast3 (PAleontologicalSTatistics).

\section{RESULTADOS}

Los resultados obtenidos permitieron 
contabilizar 1.404 individuos pertenecientes a dos órdenes, ocho familias, ocho géneros y 12 especies (Tabla 1). Se colectaron especímenes de Procambarus clarkii (Girard, 1852), especie exótica que no había sido reportada para el norte de Sinaloa, ni para el río Fuerte. También se identificaron individuos de Potimirim glabra (Kingsley, 1878), sin registro previo en la región. Las cinco especies de mayor abundancia relativa durante el periodo de estudio fueron mayor y menor diversidad, respectivamente. San José y San Blas registraron la mayor y menor dominancia, respectivamente. San Blas tuvo el mayor índice de equidad y San Miguel la de mayor riqueza de especies (Tabla 2).

La similitud entre estaciones de muestreo en función de las especies presentes y las compartidas (análisis de ordenación nMDS de Bray Curtis) se muestra en la Figura 3. El análisis nMDS indicó que la comunidad se puede

Tabla 1. Arreglo taxonómico y abundancia de los macrocrustáceos en número de organismos del río Fuerte. Table 1. Systematic arrangement and total abundance of macrocrustaceans in organism numbers from the río Fuerte.

\begin{tabular}{ccclc}
\hline $\begin{array}{c}\text { Orden } \\
2\end{array}$ & Familia & Género & \multicolumn{1}{c}{ Especie } & Abundancia \\
\hline Decapoda & Palaemonidae & Macrobrachium & \multicolumn{1}{c}{$\begin{array}{l}\text { tenellum (Smith, 1871) } \\
\text { diguetti (Bouvier, 1895) }\end{array}$} & 974 \\
& & & occidentale (Holthuis, 1950) & 11 \\
& & & americanum (Bate, 1868) & 8 \\
& & & hobbsi (Nates \& Villalobos, 1990) & 29 \\
& & & & 11 \\
& Cambaridae & Procambarus & clarkii (Girard, 1852) & 14 \\
& Atyidae & Potimirim & glabra (Kingsley, 1878) & 220 \\
& Portunidae & Callinectes & arcuatus (Ordway, 1863) & 7 \\
& Penaeidae & Litopenaeus & vanammei (Boone, 1931) & 3 \\
& Diogenidae & Clibanarius & panamensis (Stimpson, 1859) & 67 \\
& Ocypodidae & Uca & crenulata (Lockington, 1877) & 31 \\
Amphipoda & Hyalellidae & Hyalella & azteca (Saussure, 1858) & 30 \\
\hline
\end{tabular}

Macrobrachium tenellum (Smith, 1871), P. glabra, Clibanarius panamensis (Stimpson, 1859), Macrobrachium americanum y Hyalella azteca (Saussure, 1858) (Figura 2). San Blas y San José constituyeron las estaciones de muestreo con agrupar en tres grupos bien definidos (Figura 3). Esto estuvo dado principalmente por la distancia espacial de las estaciones, donde la estación El Fuerte se encuentra al norte, situada en una zona influenciada por el desagüe de la Sierra

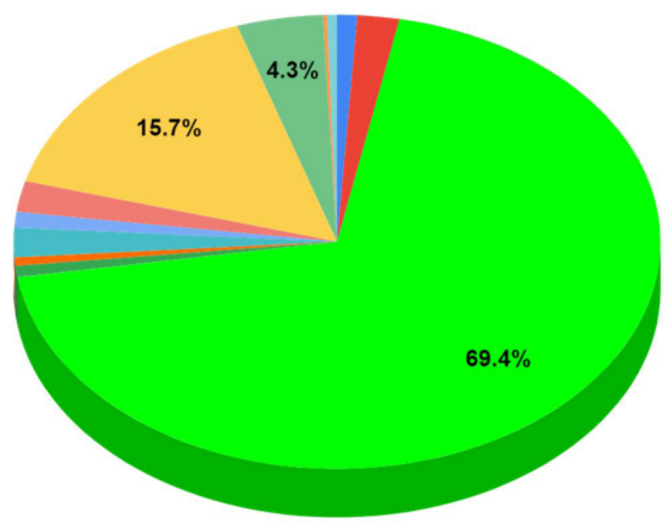

Procambarus clarkii

- Hyallela azteca

Macrobrachium tenellum

Macrobrachium diguetti

Macrobrachium occidentale

Macrobrachium americanum

Macrobrachium hobbsi

Uca crenulata

Potimirim glabra

Clibanarius panamensis

Litopenaeus vanammei

Callinectes arcuatus

Figura 2. Abundancia relativa de macrocrustáceos durante el periodo de estudio en el río Fuerte, Sinaloa, México. Figure 2. Relative abundance of macrocrustaceans during the study period in Fuerte River, Sinaloa, Mexico. 
Tabla 2. Índices ecológicos calculados por estación de muestreo durante el periodo de estudio. $\mathrm{H}$ : Diversidad Shannon-Wiener, D: Dominancia Simpson, J: Equidad Pielou, S: riqueza específica.

Table 2. Ecological indices calculated by sampling station during the study period. H: Shannon-Wiener Diversity, D: Dominance Simpson, J: Equity Pielou, S: specific richness.

\begin{tabular}{lcccc}
\hline $\begin{array}{l}\text { Estaciones de } \\
\text { muestreo }\end{array}$ & $\mathrm{H}$ & $\mathrm{D}$ & $\mathrm{J}$ & $\mathrm{S}$ \\
\hline El Fuerte (E I) & 0,951 & 0,4408 & 0,686 & 4 \\
San Blas (E II) & 1,392 & 0,3498 & 0,778 & 6 \\
San Miguel (E III) & 1,080 & 0,4112 & 0,555 & 7 \\
San José (E IV) & 0,239 & 0,8796 & 0,344 & 2 \\
Las Grullas (E V) & 0,943 & 0,4679 & 0,680 & 4 \\
\hline
\end{tabular}

Madre Occidental y la estación Las Grullas en dirección opuesta desembocando en la laguna costera de Agiabampo (Figura 1).

San Blas y San Miguel tuvieron el mayor número de especies en común. El Fuerte y San José les siguieron con orden decreciente de especies compartidas. Las Grullas no presentó especies en común con los demás sitios de colecta.

El análisis de PERMANOVA (Tabla 3), mostró que existen diferencias significativas en escala espacial-temporal $(P<0,05)$ en la abundancia de organismos entre estaciones de muestreos
Tabla 3. Análisis de varianza multivariado permutacional. Perms: permutaciones.

Table 3. Permutational multivariate analysis of variance. Perms: permutations.

\section{PERMANOVA}

\begin{tabular}{lcccccc}
\hline Variable & df & SS & MS & Pseudo-F & P(perm) & perms \\
Muestreo & 4 & 9270,1 & 2317,5 & 1,0301 & 0,4305 & 9857 \\
Estaciones & 4 & 51631 & 12908 & 5,7375 & $\mathbf{0 , 0 0 0 1}$ & 9891 \\
Res & 16 & 35996 & 2249,7 & & & \\
Total & 24 & 96897 & & & & \\
\hline
\end{tabular}

y durante el tiempo de colecta. No obstante, la prueba de Monte Carlo $(P<0,05)$ sugirió que con un aumento de muestras las diferencias observadas en algunas estaciones pudieran ser inexactas (Tabla 4).

\section{DISCUSIÓN}

La fauna carcinológica de agua dulce ha sido poco estudiada en nuestro país (VillalobosHiriart et al. (2010), y en menor grado en la zona norte del estado de Sinaloa. Las tres entidades de la República Mexicana con mayor diversidad de decápodos dulceacuícolas son Chiapas, Oaxaca y Veracruz con 45, 39 y 38 especies, respectivamente. Para Sinaloa,

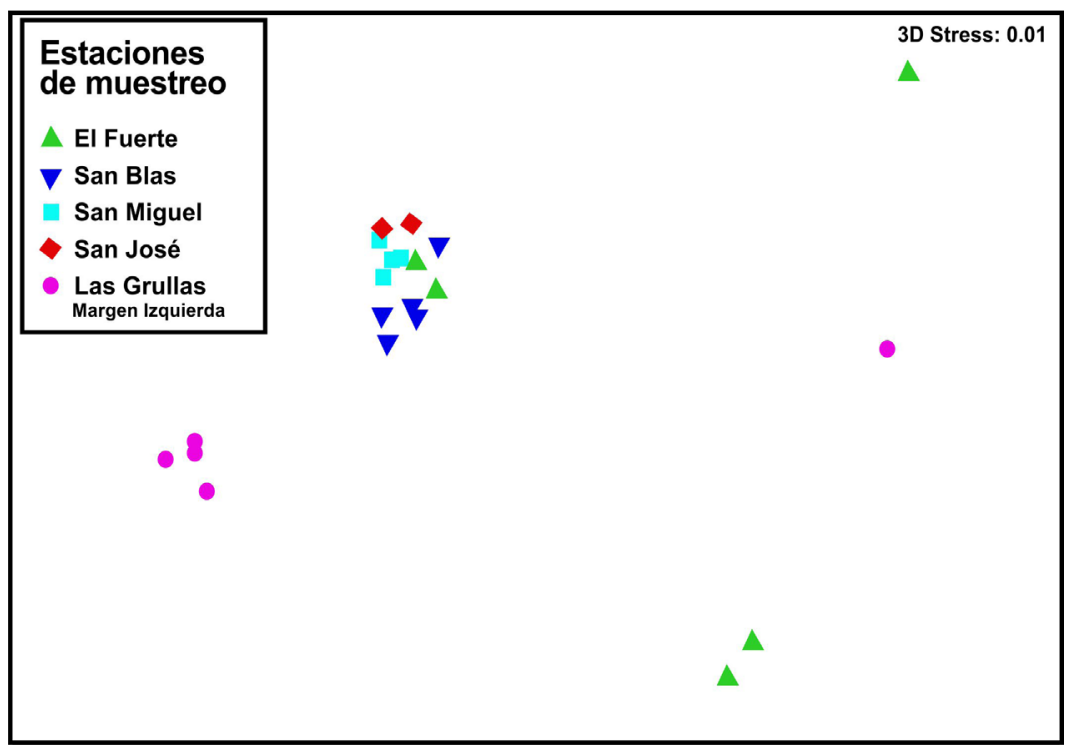

Figura 3. Análisis de Escalamiento multidimensional no métrico (nMDS B) basado en la medida de similitud de Bray-Curtis sobre los valores de composición y abundancia de las especies de crustáceos colectados en cada estación.

Figure 3. Non-metric multidimensional scaling analysis (nMDS) based on the Bray-Curtis similarity measure on the composition and abundance values of the crustacean species collected in each station. 
Tabla 4. Prueba post hoc de comparación multivariada de pares y de Monte Carlo.

Table 4. Post hoc multivariate pairwise comparison test and Monte Carlo.

\begin{tabular}{lccl}
\hline \multicolumn{4}{c}{ Prueba de pares multivariada } \\
\hline \multicolumn{1}{c}{ Grupos } & $\mathrm{t}$ & $\mathrm{P}($ perm) & perms \\
El Fuerte-San Blas & $\mathbf{1 , 7 4 0 4}$ & $\mathbf{0 , 0 4 2 2}$ & 4994 \\
El Fuerte-San Miguel & 2,2056 & $\mathbf{0 , 0 0 8 3}$ & 4872 \\
El Fuerte-San José & 2,4999 & $\mathbf{0 , 0 0 8 1}$ & 4649 \\
El Fuerte-Las Grullas & 1,6027 & $\mathbf{0 , 0 4 7 5}$ & 3976 \\
San Blas-San Miguel & 3,083 & $\mathbf{0 , 0 1 9 8}$ & 5165 \\
San Blas-San José & 3,1891 & $\mathbf{0 , 0 1 5 3}$ & 4814 \\
San Blas-Las Grullas & 2,2456 & $\mathbf{0 , 0 1 4 7}$ & 4633 \\
San Miguel-San José & 2,0931 & $\mathbf{0 , 0 3 8 8}$ & 5370 \\
San Miguel-Las Grullas & 2,8429 & $\mathbf{0 , 0 0 7 8}$ & 4628 \\
San José-Las Grullas & 3,0693 & $\mathbf{0 , 0 0 7 3}$ & 4649 \\
\hline
\end{tabular}

Rosas-Hernández (2012) reportó 13 especies, aunque previamente Álvarez et al. (1996), habían mencionado un total de 16 especies. Estas discrepancias y el bajo número de publicaciones al respecto dificultan el conocimiento acerca de la diversidad carcinológica de agua dulce en Sinaloa, especialmente, en el río Fuerte. En esta investigación se lograron identificar 12 especies de las cuales, el 91,6\% (11) correspondieron a decápodos. van der Heiden et al. (2015) reportaron seis especies de decápodos, capturados en muestreos realizados en épocas de lluvias y con mayor frecuencia en época de estiaje durante nueve años en el Río Panuco (Concordia, Sinaloa). Aparentemente el río Fuerte cuenta con mayor riqueza específica, sin embargo, de los 11 decápodos reportados en este trabajo, cuatro de ellos se consideran de ambientes salobres-marinos (Hendrickx, 1995). Además, del total antes mencionado, se registró la presencia de P. g/abra y P. clarkii, destacándose que no habían sido reportadas previamente para esta zona específica de estudio (Hernández et al., 2008). En este sentido, Villalobos-Hiriart y Álvarez (2008) indicaron que la presencia de nuevas especies podría ser frecuente en ciertas regiones de México, ya que no han sido debidamente muestreadas, incluyendo la presencia de grupos como los cangrejos de agua dulce de la familia Pseudothelphusidae, que sólo en las últimas dos décadas han sido investigados con mayor intensidad.

Por otro lado, átyidos y palemónidos, son los crustáceos más frecuentes en las porciones media y baja de las vertientes occidental y oriental del país. Un número importante de especies de estas familias, como Atya margaritacea (A. Milne-Edwards, 1864) y $M$. tenellum, son de gran importancia comercial debido a su abundancia para las zonas donde se localizan (Lorán-Nuñez et al., 2009; MartínezMayén et al., 2000; Rodríguez de la Cruz, 1965). Tales aseveraciones concuerdan con los resultados obtenidos en esta investigación, ya que el palemónido $M$. tenellum fue la especie más abundante (Tabla 1) y frecuente en las localidades de muestreo. Las especies del género Macrobrachium presentaron una distribución característica. Macrobrachium tenellum se localizó únicamente en zonas cercanas a la desembocadura del río (San Miguel, San José y Las Grullas) mientras que $M$. americanum (Bate, 1868), M. digueti (Bouvier, 1895), M. occidentale (Holthuis, 1950) y M. hobbsi (Nates \& Villalobos, 1990) sólo se hallaron en las estaciones de muestreo más alejadas a la zona costera. En ese sentido, Aguilar et al. (1998) reportaron que existen diferentes tipos de langostino de acuerdo con el nivel de salinidad que pueden tolerar. Macrobrachium tenellum tolera salinidades hasta 30\%o (Hernández et al., 2018) por lo que se encuentra en las estaciones más cercanas a la costa. En la actualidad, existen registros en los que se ha observado una tendencia de disminución de la diversidad conforme se incrementa la altitud. Sin embargo, el gradiente altitudinal observado en este trabajo no fue tan marcado como lo señalado en otras investigaciones (Mejía-Ortiz et al., 2001; Mejía-Ortiz y Álvarez, 2010). Se observó que San Blas y San Miguel presentaron mayor diversidad, lo cual coincidió con una dominancia más baja y una equidad intermedia-alta. El índice de equidad $\left(J^{\prime}\right)$ no mostró gran diferencia, y el valor más alto coincidió con el sitio que mostró el valor de diversidad más elevado (Tabla 2). Esto se debió posiblemente a que las hembras ovígeras de átyidos y palemónidos utilizan las grandes crecidas de los ríos en tiempos de lluvia para realizar sus movimientos desde las cabeceras hacia la porción baja de los ríos, con el fin de liberar sus larvas en los ambientes estuarinos donde encuentran las condiciones favorables para iniciar su desarrollo Jalihal et al., 1993; 
Rome et al., 2009). Por tal razón organismos juveniles de $M$. tenellum fueron observados en San Miguel y San José, ya que la temporada de lluvias recién se iniciaba en la región Noroeste de México. En la última estación de muestreo (Las Grullas) se encontraron ocypodidos, peneidos, paguroideos y portunidos característicos de ambientes oligohalinos y costeros (EscobarBriones et al., 1999; Sánchez y Barba 2005), siendo la salinidad en esta zona de $16 \%$ durante el periodo de muestreo. Este valor es elevado con respecto al resto de las estaciones de muestreo, razón por la cual no hubo similitud de especies con los otros sectores del río.

\section{AGRADECIMIENTOS}

Se agradece a dos revisores anónimos sus observaciones para mejorar el presente trabajo, también a la Dra. Viridiana Peraza Gómez y al Dr. Osmar Araujo por su apoyo en los análisis aplicados y a estudiantes de diversas asignaturas de los PE de Ingeniería ambiental y Licenciatura en Biología de la Universidad Autónoma de Occidente U.R. Los Mochis, por su ayuda en la realización de los muestreos.

\section{REFERENCIAS}

Aguilar, M., Díaz, F. \& Buckle, L. F. (1998). The effect of salinity on oxygen consumption and osmoregulation of Macrobrachium tenellum. Marine Freshwater Behaviour and Physiology, 31, 105-113.

Álvarez, F., Villalobos, J. L. y Lira, E. (1996). Decapoda. En: Llorente Bousquets, J. E., Morrone, J. J., Yáñez Ordóñez, O. y Vargas Fernández, I. (Eds.). Biodiversidad, taxonomía y biogeografía de artrópodos de México: hacia una síntesis de su conocimiento (pp.103-129). Conabio. México, D.F.

Álvarez, F., Villalobos, J. L., Hendrickx, M. E., Escobar-Briones, E., Rodríguez-Almaraz, G. y Campos, E. (2014). Biodiversidad de crustáceos decápodos (Crustacea: Decapoda) en México. Revista Mexicana de Biodiversidad, Supl. 85, 208-219.
Araujo-Leyva, O., Rodríguez-Villanueva, L. V. \& Macías-Zamora, J. V. (2020). Biodiversity of polychaetous annelids in Bahía de Todos Santos, Baja California México. Zoosymposia 19, 051-071

Baev, P. V \& Penev, L. D. (1995). BIODIV: program for calculating biological diversity parameters, similarity, niche overlap, and cluster analysis. Versión 5.1. Pensoft.

Bjerkeng, B. (2000). Carotenoid pigmentation of salmonid fishes - recent progress. In: Cruz -Suárez, L. E., Ricque-Marie, D., Tapia Salazar, M. Olvera-Novoa, M. A. y Civera-Cerecedo, R., (Eds.). Avances en Nutrición Acuícola V. Memorias del V Simposium Internacional de Nutrición Acuícola. (pp. 19-22). Mérida, Yucatán.

CONAGUA. (2016). Atlas del Agua en México. Comisión Nacional del Agua. Subdirección General de Planeación. SINA. México D.F., México.

Escobar-Briones, E., Álvarez, F. \& Salgado, G. (1999). Discapseudes holthisi Crustacea: Tanaidacea) as an intermediate host of Caballerohynchus lamothei (Acanthocephala: Cavasomidae). Journal of Parasitology, 85, 134 137.

García-Madrigal, M. S., Villalobos-Hiriart, J. L., Álvarez, F. y Bastida-Zavala, R. (2012). Estado del conocimiento de los crustáceos de México. Ciencia y Mar, 46, 43-62.

Hendrickx, M. E. (1995). Camarones. En: Fischer, W., Krupp, F., Schneider, W., Sommer, C., Carpenter K. E. y Niem, V. H. (Eds.). Guía FAO para la identificación de especies para los fines de pesca. Pacífico centro- oriental. Vol. 1. Plantas e invertebrados. (pp. 417-537). Roma: FAO.

Hernández, L., Maeda-Martínez, A. M., RuizCampos, G., Rodríguez-Almaraz, G., AlonzoRojo, F. \& Sainz, J. C. (2008). Geographic expansion of the invasive red crayfish Procambarus clarkii (Girard, 1852) (Crustacea: Decapoda) in Mexico. Biological Invasions 10, 977-984. 
Hernández-Sandoval, P., Díaz, F., Re-Araujo, D., López-Sánchez, J. A., Martínez-Valenzuela, M. C., García-Guerrero, M. U. \& Rosas, C. (2018). Thermal preference, critical thermal limits, oxygen routine consumption and active metabolic scope of Macrobrachium tenellum (Smith, 1871) maintained at different acclimation temperatures. Latin American Journal of Aquatic Research, 46, 558-569.

INEGI (2017). Anuario estadístico y geográfico de Sinaloa 2017. México: Inegi.

Jalihal, D. K., Sankolli, K. N. \& Shenoy, S. (1993). Evolution of larval development patterns and the process of freshwaterization in the prawn genus Macrobrachium Bate, 1868 (Decapoda: Palaemonidae). Crustaceana, 65, 365-376.

Lorán-Nuñez, R. M., Valdéz-Guzmán, A. J. y Martínez-Isunza, F. R. (2009). Estudio biológico-pesquero del "burro" Atyas cabra en el río de los Pescados y río Actopan, Veracruz, México. Ciencia Pesquera, 17, 5-16.

Magurran, A. E. (1988). Ecological diversity and its measurement. New Jersey: Princeton University Press.

Marmol, Z., Páez, G., Rincón, M., Araujo, K., Aiello, C., Chandler, C. y Gutiérrez, E. (2011). Quitina y quitosano polímeros amigables. Una revisión de sus aplicaciones. Revista Tecnocientífica URU, 1, 53-58.

Martin, J. W. \& Davis, G. E. (2001). An updated classification of the recent Crustacea. Natural History Museum of Los Angeles County Science Series, 39, 1-124.

Martínez-Mayén, M., Román-Contreras, R., Rocha-Ramírez, A. \& Cházaro-Olvera S. (2000). Relative growth of Atya margaritacea A. Milne-Edwards, 1864 (Decapoda, Atyidae) from the southern Pacific coast of Mexico. Crustaceana, 73, 525-534.

Mejía-Ortiz, L. M., Álvarez, F., Román, R. \& Viccon-Pale, J. A. (2001). Fecundity and distribution of freshwater prawns of the genus Macrobrachium in the Huitzilapan River, Veracruz, Mexico. Crustaceana, 74, 69-77.

Mejía-Ortiz, L. M. \& Álvarez, F. (2010). Seasonal patterns in the distribution of three species of freshwater shrimp Macrobrachium spp., along an altitudinal river gradient. Crustaceana, 3, 385-397.

Moreno, C. E. (2001). Métodos para medir la Biodiversidad. M\&T-Manuales y Tesis SEA, Zaragoza.

Orrantia Verduzco, C. A. (2019). Rectificación de cauce de río Fuerte, con aprovechamiento del material pétreo; en el municipio de El Fuerte, Sinaloa. Manifiesto de Impacto AmbientalParticular. Los Mochis, Sinaloa.

Rodríguez de la Cruz, M. C. (1965). Contribución al conocimiento de los Palemónidos de México II. Palemónidos del Atlántico y vertiente oriental de México con descripción de dos especies nuevas. Anales del Instituto Nacional de Investigaciones Biológico Pesqueras, 1, 70-112.

Rodríguez-Almaraz, G., Ortega-Vidales, V. y Treviño-Flores, J. A. (2014). Macrocrustáceos del Parque Nacional Cumbres de Monterrey, México: distribución y estado de conservación. Revista Mexicana de Biodiversidad, 85, 276-293.

Rome, N. E., Conner, S. L. \& Bauer, R. T. (2009) Delivery of hatching larvae to estuaries by an amphidromous river shrimp: tests of hypothesis based on larval moulting and distribution. FreshwaterBiology, 54, 1924-1932.

Rosas-Hernández, M. I. (2012). Distribución de los crustáceos decápodos de agua dulce de México. Tesis de licenciatura. Universidad Nacional Autónoma De México. Facultad de ciencias. D.F. México.

Sánchez, A. J. y Barba, E. (2005). Biodiversidad de Tabasco. En: Bueno, J., Álvarez, F. y Santiago, S. (Eds.). Biodiversidad del estado de Tabasco (pp. 1-16). Instituto de Biología, UNAM / CONABIO, D. F. México. 
Simões N., Mascaró M., Ordoñez U. y Ardisson, P. L. (2004). Crustáceos. En: Duran-García, R. y Méndez-González, M.E. (Eds) Biodiversidad y Desarrollo Humano en Yucatán. (pp223-225). CICY, PPD-FMAM, CONABIO, SEDUMA. Yucatan. México.

van der Heiden, A. M., Plascencia-González, E. G., Villalobos-Hiriart, J. L. y Espinosa-Pérez, H. S. (2015). Los peces y macrocrustáceos decápodos de la cuenca media del río Pánuco en la Sierra Madre Occidental del sur de Sinaloa, México. En: González Elizondo, M. S., González Elizondo, M. y Cortés Montaño, C. (Eds.). Biodiversidad y Paisaje de la Sierra Madre Occidental (pp. 106136). México: Instituto Politécnico NacionalCONABIO.

Villalobos-Hiriart, J. L., Díaz-Barriga, A. C. y Lira-Fernández, E. (1993). Los crustáceos de agua dulce de México. En: Gío-Argáez, R. y López-Ochoterena, E. (Eds.). Diversidad biológica en México. Revista de la Sociedad Mexicana de Historia Natural, volumen especial 64: 267-290.

Villalobos-Hiriart, J. L. y Álvarez, F. (2008). Los cangrejos de agua dulce de la familia Pseudothelphusidae (Decapoda: Brachyura: Eubrachyura) de México. Con un apéndice de las especies citadas para América hasta el 2006. En: Álvarez, F. y Rodríguez, G. (Eds). Crustáceos de México: estado actual de su conocimiento (pp. 239-299). PROMEP-UANL, Secretaría de Educación Pública/Dirección General de Publicaciones, Universidad Autónoma de Nuevo León, Monterrey.

Villalobos-Hiriart, J. L., Álvarez-Noguera, F., Hernández, C., De La Lanza-Espino, G. y González-Mora, I. D. (2010). Crustáceos decápodos de las cuencas Copalita, Zimatán y Coyula, en Oaxaca, México. Revista Mexicana de Biodiversidad, 81, 99-111. 